\title{
Síntese, caracterização e propriedades de sistemas contendo óxidos de lantânio, cério e níquel obtidos pelo método dos precursores poliméricos
}

\author{
(Synthesis, characterization and properties of the systems containing \\ lanthanum, cerium and nickel oxides obtained by polymeric precursor)
}

\author{
D. M. A. Melo, V. G. Oliveira, A. M. G. Pedrosa, P. M. Pimentel, D. K. S. Gomes, J. D. G. Fernandes \\ Departamento de Química, Universidade Federal do Rio Grande do Norte, C.P. 1662, Natal, RN 59078-970
}

\begin{abstract}
Resumo
No presente trabalho estuda-se a síntese, caracterização e propriedades fotoluminescentes de sistemas contendo óxidos de lantânio, cério e níquel. Estes materiais foram preparados pelo método dos precursores poliméricos, calcinados na faixa de temperatura de 300 a $800{ }^{\circ} \mathrm{C}$ e então caracterizados por análise $\mathrm{CHN}$, análise termogravimétrica, infravermelho com transformada de Fourier e difração de raios X. Os resultados revelaram a formação de fase cristalina em $800{ }^{\circ} \mathrm{C}$. Os espectros fotoluminescentes dos sistemas desordenados foram registrados a temperatura ambiente. O sistema de lantânio, cério e níquel apresentou forte intensidade fotoluminescente, centrado em $599 \mathrm{~nm}$, quando excitado a $488 \mathrm{~nm}$.

Palavras-chave: óxidos de lantânio, cério e níquel, fotoluminescência, precursor polimérico.
\end{abstract}

Abstract

The synthesis, characterization and photoluminescence property of the systems containing lanthanum, cerium and nickel oxides were studied. These materials were prepared by the polymeric precursors, calcined at temperatures ranging from 300 to $800{ }^{\circ} \mathrm{C}$. Afterwards they were characterized by CHN analysis, thermogravimetric analysis, Fourier-transform infrared spectroscopy and $X$ ray diffraction. The results revealed the formation of the crystalline phases at $800^{\circ} \mathrm{C}$. The photoluminescence spectra of disordered systems have been observed at room temperature. The lanthanum, cerium and nickel system presented strong photoluminescence intensity centered at $599 \mathrm{~nm}$ when excited to $488 \mathrm{~nm}$.

Keywords: lanthanum, cerium and nickel oxides, photoluminescence, polymeric precursor.

\section{INTRODUÇÃO}

Sistemas contendo óxidos de níquel e de terras raras têm mostrado interessantes propriedades ópticas, catalíticas e eletrônicas [1-3]. Desde os primeiros relatos de fotoluminescência (FL) em silício poroso, há um crescente interesse no estudo das propriedades FL em matérias nanoestruturados e desordenados [4]. Recentemente, foi observado que os óxidos desordenados exibem fortes propriedades luminescentes na região do visível [5-7]. No estado cristalino, estes materiais são conhecidos por apresentar descontinuidades maiores que $3 \mathrm{eV}$, sugerindo que o espectro FL não tem sua origem nas transições eletrônicas, como ocorre com semicondutores em geral [8]. A produção de cerâmica de alta performance apresentando tais propriedades tem incentivado a pesquisa, usando-se novas rotas de síntese para pós. Os sistemas contendo óxidos de lantânio, cério e níquel podem ser produzidos por vários métodos, incluindo rota cerâmica convencional, processamento solgel, co-precipitação, decomposição de nitratos, métodos hidrotérmicos, entre outros [9-11]. A maioria das técnicas mencionadas geralmente requer alta temperatura, fluxo de oxigênio e longos períodos de calcinação (cerca de $16 \mathrm{~h}$ ) para obtenção do material homogêneo.

No presente estudo, sistemas contendo óxidos de lantânio, cério e níquel foram sintetizados utilizando o método dos precursores poliméricos [12]. Este método permite um considerável controle estequiométrico, reprodutibilidade e homogeneidade quando comparado aos métodos tradicionais. Estas características são relevantes no que diz respeito à determinação das propriedades catalíticas, eletrônicas, magnéticas e ópticas deste material. O sistema contendo óxidos de lantânio, cério e níquel foi escolhido devido à expectativa de que a substituição parcial do lantânio pelo cério na estrutura perovsquita provoque um grau de desordem no sistema e consequentemente afete suas propriedades fotoluminescentes. Vários trabalhos na literatura têm discutido as propriedades fotoluminescentes de sistemas similares a estes $[13,14,16]$.

\section{PROCEDIMENTO EXPERIMENTAL}

Os sistemas contendo óxidos de lantânio, cério e níquel (S1) e óxidos de cério e níquel (S2) foram preparados pelo método dos precursores poliméricos [12]. Como materiais de partida foram usados nitrato de lantânio (Aldrich, 99\%), 
nitrato de níquel (Vetec, 97\%), cloreto de cério (Aldrich, 99\%), ácido cítrico (Vetec, 99,5\%) e etileno glicol (Vetec, 99\%). Os sistemas S1 e S2 foram preparados em três etapas: (1) preparação dos citratos; (2) adição e mistura de todos os cátions metálicos; (3) polimerização do sistema. O citrato de níquel foi preparado a partir da dissolução do nitrato de níquel sólido em solução aquosa de ácido cítrico (AC), sob agitação a $60-70{ }^{\circ} \mathrm{C}$ por $1 \mathrm{~h}$ (proporção molar Ni:AC = 1:3). Em seguida, adicionaram-se ao citrato preparado os nitratos de lantânio e cloreto de cério (na síntese do sistema S1) ou apenas o cloreto de cério (na síntese do sistema S2). Os produtos resultantes foram homogeneizados a $90{ }^{\circ} \mathrm{C}$. Logo após, o etilenoglicol foi adicionado numa proporção de 40:60 (ácido cítrico: etilenoglicol) e a temperatura foi mantida constante durante $2 \mathrm{~h}$ resultando em uma resina. Esta resina foi pirolisada a $300{ }^{\circ} \mathrm{C}$ por $2 \mathrm{~h}$. Os materiais obtidos foram calcinados a $800{ }^{\circ} \mathrm{C}$ por $4 \mathrm{~h}$ sob velocidade de aquecimento de $5{ }^{\circ} \mathrm{C} \mathrm{min}{ }^{-1}$. Os sistemas $\mathrm{S} 1$ e S2 foram preparados com base nas seguintes composições teóricas: $\mathrm{La}_{0,9} \mathrm{Ce}_{0,1} \mathrm{NiO}_{3} \mathrm{e}$ $\mathrm{CeO}_{2}-\mathrm{NiO}\left(\mathrm{CeNiO}_{3}\right)$.

Os teores de carbono, hidrogênio e nitrogênio ( $\mathrm{CHN})$ foram determinados em um analisador da Perkin Elmer CHN-2400 com o objetivo de confirmar a presença de resíduos orgânicos, após a decomposição térmica da solução polimérica. As curvas termogravimétricas (TG) foram obtidas em uma balança da Perkin Elmer TGA-7 sob atmosfera de ar e uma razão de aquecimento de $5{ }^{\circ} \mathrm{C}$ $\min ^{-1}$. A presença de matéria orgânica remanescente após calcinação foi analisada por espectroscopia de absorção na região do infravermelho (FTIR) usando pastilhas de $\mathrm{KBr}$ em um instrumento ABB MB-104. Os óxidos calcinados foram analisados por difração de raios X (DRX) usando radiação $\mathrm{CuK}_{\alpha}$ em um equipamento Shimadzu XRD-6000. Os dados foram coletados em um intervalo de variação angular entre 5 e $75^{\circ}$. Os espectros fotoluminescentes foram obtidos em um espectrômetro Jobin-Yvon modelo Raman U1000, tendo como detector uma fotomultiplicadora RCA refrigerada por um sistema Peltier. As amostras foram excitadas utilizando um laser de argônio $(488 \mathrm{~nm})$. Todas as medidas foram realizadas na temperatura ambiente.

\section{RESULTADOS E DISCUSSÃO}

A Fig. 1 mostra as curvas termogravimétricas correspondentes à decomposição do material pirolisado a $300{ }^{\circ} \mathrm{C}$ dos sistemas $\mathrm{S} 1$ e S2. A decomposição térmica destes sistemas ocorre em três patamares e se estabiliza entre 720$800{ }^{\circ} \mathrm{C}$. A perda de massa observada entre 30 e $800{ }^{\circ} \mathrm{C}$ é atribuída a decomposição de matéria orgânica remanescente do processo de síntese. As curvas termogravimétricas dos sistemas óxidos S1 e S2 calcinados a $800{ }^{\circ} \mathrm{C}$ (Figura não mostrada) quase não apresenta matéria orgânica residual confirmando a estabilidade da fase óxido obtida.

Os espectros de absorção na região do infravermelho para os sistemas S1 e S2 tratados a 300 e $800^{\circ} \mathrm{C}$ são mostrados na Fig. 2. As vibrações em $1538-1350 \mathrm{~cm}^{-1}$ foram relacionadas à coordenação dos cátions $\mathrm{Ni}^{3+}$ pelos grupos carboxílicos na

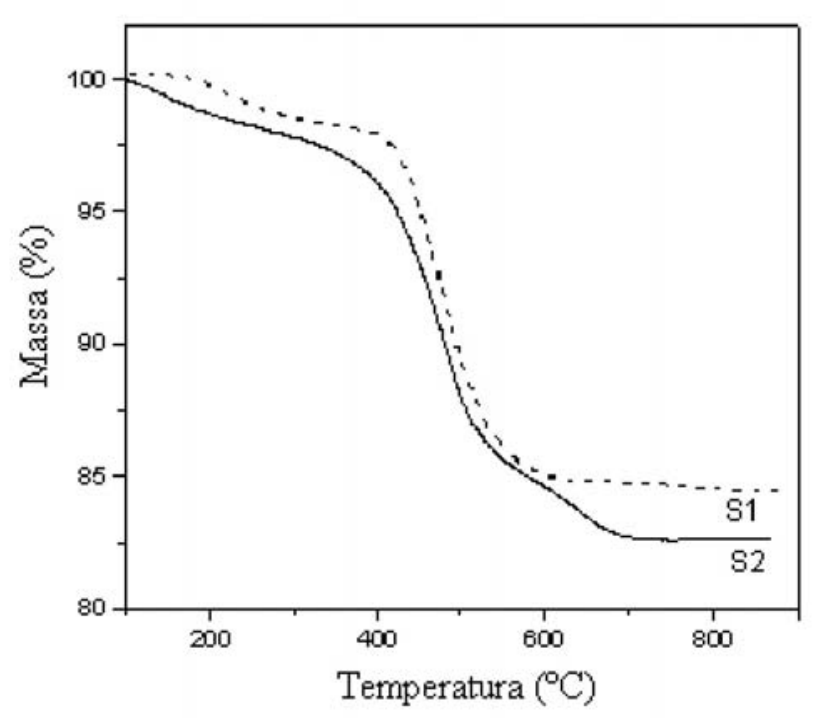

Figura 1: Curvas termogravimétricas dos sistemas com La, Ce e Ni (S1) e com Ce e $\mathrm{Ni}(\mathrm{S} 2)$ calcinadas em $300{ }^{\circ} \mathrm{C}$.

[Figure 1: Thermogravimetric curves of La, Ce and Ni compounds (S1) and Ce and Ni compounds (S2) after calcination at $300^{\circ} \mathrm{C}$.]

Tabela I - Resultados de análise elementar CHN (\%) para as amostras S1 e S2.

[Table I - CHN analysis of the S1 and S2 compounds.]

\begin{tabular}{ccccc}
\hline & $\begin{array}{c}\text { Temperatura } \\
\left({ }^{\circ} \mathrm{C}\right)\end{array}$ & $\begin{array}{c}\mathrm{C} \\
(\%)\end{array}$ & $\begin{array}{c}\mathrm{H} \\
(\%)\end{array}$ & $\begin{array}{c}\mathrm{N} \\
(\%)\end{array}$ \\
\hline \multirow{2}{*}{$\mathrm{S} 1$} & 300 & 0,37 & 0,15 & 0,06 \\
& 800 & 0,07 & 0,09 & 0,01 \\
\multirow{2}{*}{$\mathrm{S} 2$} & 300 & 0,41 & 0,20 & 0,06 \\
& 800 & 0,05 & 0,08 & 0,03 \\
\hline
\end{tabular}

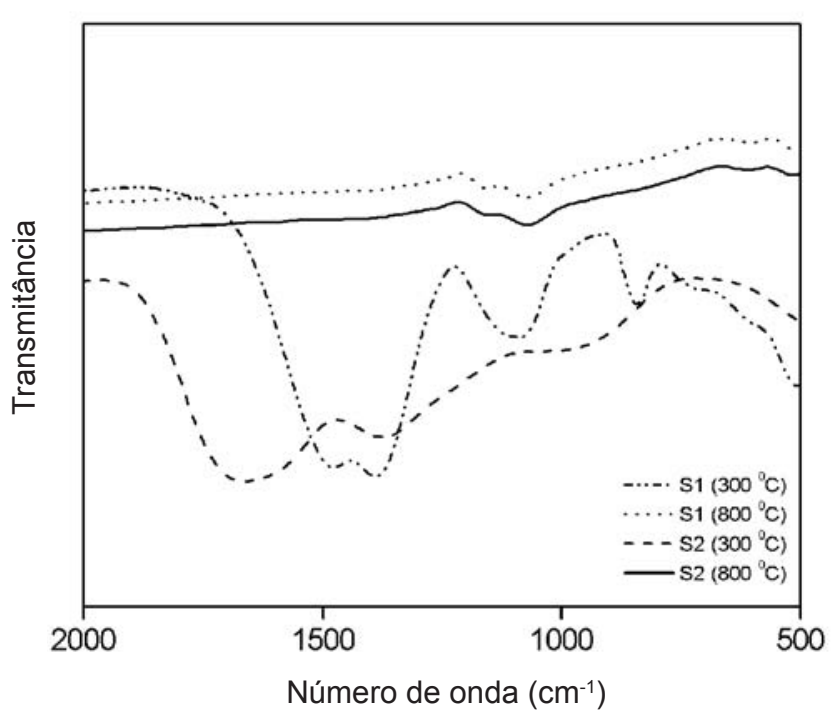

Figura 2: Espectros FTIR dos sistemas S1 e S2 calcinados em $300^{\circ} \mathrm{C}$ e $800^{\circ} \mathrm{C}$.

[Figure 2: FTIR spectra of S1 and S2 compounds after calcination at $300^{\circ} \mathrm{C}$ and $800^{\circ} \mathrm{C}$.] 
forma de um complexo bidentado [15]. Os espectros FTIR dos pós-calcinados a $800{ }^{\circ} \mathrm{C}$ confirmaram a decomposição da matéria orgânica pelo desaparecimento das principais bandas referentes aos grupos orgânicos provenientes do processo de síntese. Os resultados da análise elementar CHN dos sistemas S1 e S2 como função da temperatura de calcinação são listados na Tabela I. Os teores dos elementos decrescem quando a temperatura de calcinação aumenta, claramente indicando o processo contínuo da eliminação de material residual.

Os difratogramas de raios $\mathrm{X}$ dos sistemas $\mathrm{S} 1$ e $\mathrm{S} 2$ tratados a $300^{\circ} \mathrm{C}$ (Figura não mostrada) não apresentam formação de fases cristalinas. Os difratogramas de raios $\mathrm{X}$ dos pós $\mathrm{S} 1$ e S2 calcinados a $800{ }^{\circ} \mathrm{C}$ (Fig. 3) sugerem a formação de pós altamente cristalinos. Diversas fases foram observadas nestes compostos, o que sugere que a cristalinidade não é função da estrutura monofásica. Picos referentes a óxidos de níquel e niquelatos de lantânio (fase perovsquita) e óxidos de lantânio foram observados no sistema S1, Fig. 3. O sistema S2 apresentou estrutura cristalina, mas nenhuma evidência existe sobre a formação da fase perovsquita. Por outro lado, as formações dos óxidos de níquel e cério estão presentes com boa definição.

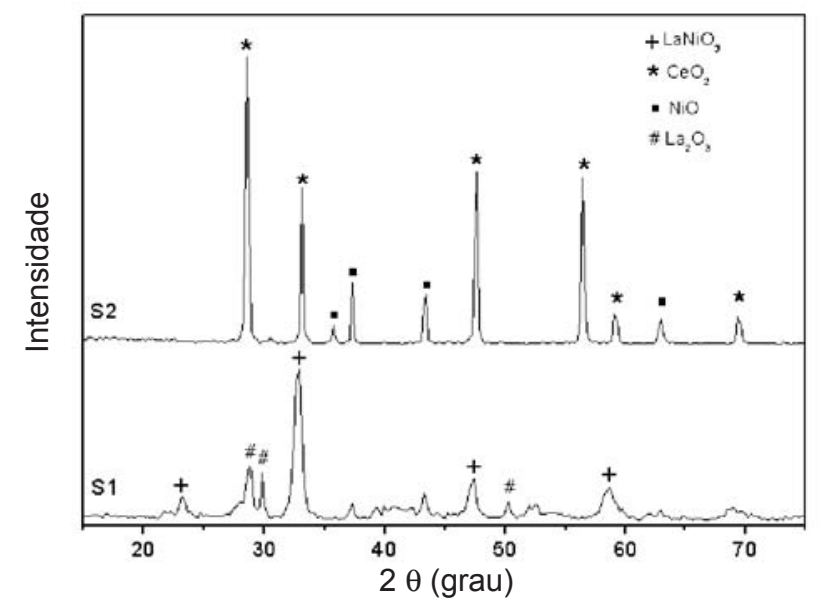

Figura 3: Difratogramas de raios X dos sistemas S1 e S2 calcinados em $800^{\circ} \mathrm{C}$.

[Figure 3: X-ray diffraction patterns of S1 and S2 compounds after calcination at $800^{\circ} \mathrm{C}$.]

A Fig. 4 mostra o espectro fotoluminescente (FL) dos sistemas S1 e S2 amorfos (calcinados a $300{ }^{\circ} \mathrm{C}$ ) obtidos com um comprimento de onda de excitação de $480 \mathrm{~nm}$ e à temperatura ambiente. $\mathrm{O}$ espectro FL, apresentado pelo sistema S2 não apresentou nenhuma emissão na região visível. No entanto, para o sistema S1, uma banda larga de intensidade máxima centrada em torno de $559 \mathrm{~nm}$ é um indicativo de intensa emissão fotoluminescente. Este efeito é atribuído à desordem estrutural do meio, e a existência de oxigênio não ligado na estrutura formadora do óxido durante o processo de cristalização da estrutura perovsquita. A intensidade FL também pode estar relacionada ao grau de desordem no sistema $\mathrm{S} 1$, causado pela pequena substituição do cério dentro do niquelato de lantânio. Espectros FL dos óxidos

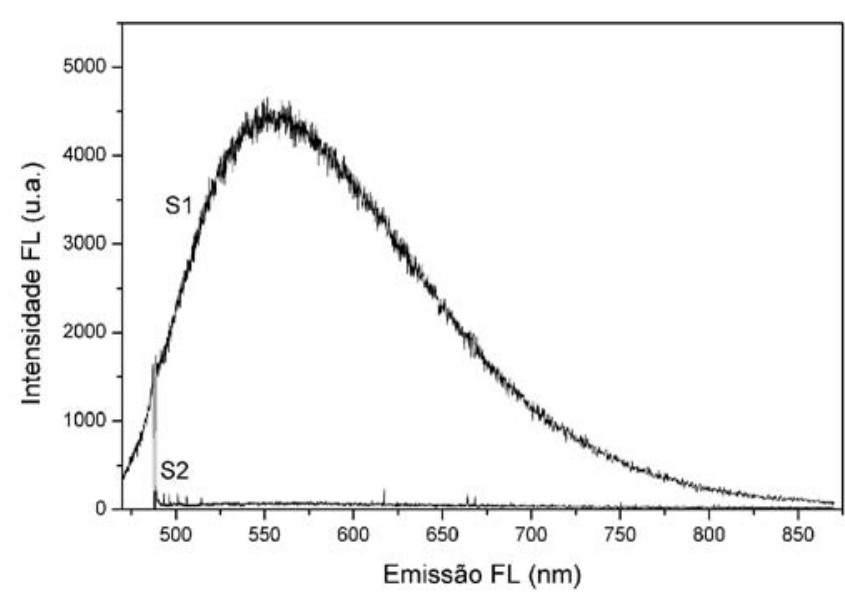

Figura 4: Espectros de fotoluminescência dos sistemas S1 e S2 calcinados em $300{ }^{\circ} \mathrm{C}$.

[Figure 4: Photoluminescence spectra of S1 and S2 compounds after calcination at $300^{\circ} \mathrm{C}$.]

contendo terras-raras em sistemas desordenados e obtidos a temperatura ambiente têm sido observados por alguns autores [15]. Fernandes e colaboradores observaram que o niquelato de lantânio amorfo produz uma luminescência relacionada com a mudança de $\mathrm{Ni}^{3+}$ para $\mathrm{Ni}^{2+}$ a qual deve ser compensada pela carga negativa do oxigênio (NBO) [15-16].

\section{CONCLUSÕES}

Sistemas contendo óxidos de lantânio, cério e níquel (S1) e óxidos de cério e níquel (S2) foram sintetizados pelo método dos precursores poliméricos. Os resultados mostraram que os materiais cristalizam formando uma mistura de óxidos. Estudo sobre a decomposição térmica dos sistemas S1 e S2 calcinados a $300{ }^{\circ} \mathrm{C}$ revelou que a completa decomposição da matéria orgânica remanescente do processo de síntese ocorre a aproximadamente $720^{\circ} \mathrm{C}$. A fotoluminescência é função da formação de vacâncias em estado amorfo e pode ser provocada por uma mistura de óxidos responsáveis pela desordem do material.

\section{AGRADECIMENTOS}

Ao Conselho Nacional de Desenvolvimento Científico e Tecnológico (CNPq) e ao Programa de Doutorado em Engenharia e Ciência dos Materiais da UFRN.

\section{REFERÊNCIAS}

[1] K. T. Jacob, K. P. Jayadevam, R. M. Mallya, Y. Waseda, Adv. Mater. 12 (2000) 440.

[2] K. Omata, T. Takada, S. Kasahara, M. Yamada, Appl. Catal. A: Gen. 146 (1996) 255.

[3] A. M. G. Pedrosa, M. J. B. Souza, D. M. A. Melo, A. S. Araujo, L. B. Zinner, J. D. G. Fernandes, A. E. Martinelli, Solid State Sci. 5 (2003) 725.

[4] L. T. Canham, Appl. Phys. Lett. 57 (1990)1046. 
[5] W. S. Chao, E. Hamada, K. Takayanagi, J. Appl. Phys. 81 (1997) 3000.

[6] P. S. Pizani, E. R. Leite, F. M. Pontes, E. C. Paris, J. A. Varela, Appl. Phys.Lett. 77 (2000) 324.

[7] E. R. Leite, L. P. S. Santos, N. L. V. Carneiro, E. Longo, C. A. Paskocimas, J. A. Varela, C. E. M. Campos, P. S. Pizani, Appl. Phys. Lett. 78 (2001) 2148.

[8] A. G. Cullins, L. T. Caham, P. D. Calcott, J. Appl. Phys. 82 (1997) 909.

[9] A. K. Norman, M. A. Morris, J. Mater. Proc. Technol. 92 (1999) 91.

[10] J. K. Vassilion, M. Hornbostel, R. Ziebarth, F. J. Disalvo, J. Solid State Chem. 81 (1989) 208.

[11] P. Lacorre, J. B. Torrance, J. Pannetier, A. I. Nazzal,
P. W. Wang, T. C. Huang, J. Solid State Chem. 91 (1991) 225.

[12] M. Pechini, U. S. Patent 3.330.697 (1967).

[13] T. Ding, W. T. Zheng, H. W. Tian, J. F. Zang, Z.

D. Zhao, S. S. Yu, X. T. Li, F. L. Meng, Y. M. Wang, X. G. Kong, Solid State Comm. 132 (2004) 815. [14] A. M. G. Pedrosa, J. E. C. Silva, P. M. Pimentel, D. M. A. Melo, F. R. G. Silva, J. Alloys Comp. 374 (2004) 223.

[15] J. D. G. Fernandes, Tese de Doutoramento, UFRN (2002).

[16] Z. R. Silva, J. D. G. Fernandes, D. M. A. Melo, C. Alves Jr., E. R. Leite, C. A. Paskocimas, E. Longo, M. I. B. Bernardi, Mater. Lett. 56 (2002) 232.

(Rec. 10/11/2005, Rev. 19/07/2006, 09/08/2006, Ac. 22/09/2006) 